\title{
Patterns of opioid prescription post ureteroscopy among members of the Endourological Society
}

\author{
Mohannad A. Awad ${ }^{1,2}$, David W. Sobel ${ }^{3}$, Ben H. Chew ${ }^{4}$, Benjamin N. Breyer ${ }^{5}$, Mark K. Plante ${ }^{1}$, \\ Kevan M. Sternberg ${ }^{1}$
}

${ }^{1}$ Department of Surgery, Division of Urology, University of Vermont Medical Center, Burlington, VT, USA; ${ }^{2}$ Department of Surgery, King Abdulaziz University, Rabigh, Saudi Arabia; ${ }^{3}$ The Minimally Invasive Urology Institute at the Mariam Hospital, Warren Alpert Medical School of Brown University, Providence, RI, USA; ${ }^{4}$ Department of Urologic Sciences, Faculty of Medicine, University of British Columbia, Vancouver, Canada; ${ }^{5}$ Department of Urology, University of California San Francisco, San Francisco, CA, USA

Contributions: (I) Conception and design: MA Awad, DW Sobel, BN Breyer, MK Plante, KM Sternberg; (II) Administrative support: BH Chew; (III) Provision of study materials or patients: MA Awad; (IV) Collection and assembly of data: MA Awad; (V) Data analysis and interpretation: MA Awad, DW Sobel; (VI) Manuscript writing: All authors; (VII) Final approval of manuscript: All authors.

Correspondence to: Mohannad A. Awad, MD, MAS. Department of Surgery, Division of Urology, University of Vermont Medical Center, EP5 Urology, 111 Colchester Avenue, Burlington, VT 05401, USA. Email: Mohannad.awad@uvmhealth.org.

Background: Postoperative opioid prescribing has been linked with persistent opioid use. Ureteroscopy (URS) is a common urologic procedure and a potential area to focus on opioid reduction. We aim to characterize international practice patterns of opioid prescribing post URS and what measures may decrease the need for opioid prescription.

Methods: We developed a survey directed to members of the Endourological Society. The survey queried the frequency of opioid prescribing post URS, challenges when opioids are not prescribed, and measures thought to reduce the need for opioids.

Results: We received 159 responses with the majority reported practicing urology for $>20$ years $(37.1 \%)$, and performing 10-20 ureteroscopies/month (45.3\%). Forty-one percent were from the United States (US) and Canada. Sixty-six percent completed a fellowship, 84\% in endourology. Twenty-six percent prescribe opioids more than half the time and the majority do so less than $10 \%$ of the time $(61.6 \%)$. Thirty-eight percent had no challenges when opioids were omitted. Measures felt to decrease the need for opioids were preoperative counseling, nonsteroidal anti-inflammatory drugs use, and use of adjunct medications. After adjusting for location and type of practice, endourology fellowship completion, years of practice, and number of ureteroscopies/month, we found that respondents from the US and Canada were more likely to prescribe opioids more than half the time post URS compared to respondents from the rest of the world [odds ratio (OR): 87.5, $\mathrm{P}<0.001,95 \%$ confidence interval (CI): 17.3-443.5].

Conclusions: Despite proven feasibility of non-opioid pathway, nearly one-quarter of participants in our survey prescribe opioids $>50 \%$ of the time post URS. Most important factors felt to reduce opioid prescription post URS were preoperative counseling, nonsteroidal anti-inflammatory drugs use. US and Canadian urologists were more likely to prescribe opioids $>50 \%$ of the time post URS compared to the rest of the world. We believe best practice guidelines should be considered by the American and Canadian Urological Associations to address post URS opioid prescribing.

Keywords: Ureteroscopy (URS); opioids; postoperative; prescription; endourology

Submitted Jul 27, 2020. Accepted for publication Nov 02, 2020.

doi: $10.21037 /$ tau-20-1121

View this article at: http://dx.doi.org/10.21037/tau-20-1121 


\section{Introduction}

The impact of the opioid epidemic in the United States (US) is clear and research continues to focus on characterizing the role of opioid prescribing patterns to better identify ways of limiting this contributory factor. The use of opioid medications in the post-surgical setting has been shown to be an important factor and often, this may be a patient's initial exposure. There is a reported $6 \%$ incidence of persistent opioid use among opioid-naïve patients in this setting (1). Over-prescription of opioid medications is common after urologic procedures and variation in opioid prescription patterns exists after these procedures nationally $(2,3)$. In addition, patients with kidney stones may have a higher risk for long-term opioid use than non-stone formers (4).

The management of pain and stent symptoms post ureteroscopy (URS) have received particular attention partly due to the increasing incidence of stone disease (5). Non-steroidal medications have been shown to effectively manage symptoms of post-endoscopic procedural pain $(6,7)$. In addition, single-center experiences have shown the feasibility of a non-opioid approach after endourologic intervention for urolithiasis (8-10). An expert panel from the US recently recommended $0-5$ and $0-10$ Oxycodone $5 \mathrm{mg}$ pills to patients undergoing URS with lithotripsy without and with stent placement, respectively (11). The European Association of Urology (EAU) recommends NSAIDS as the first-line treatment for post URS pain control, reserving opioids for refractory pain (12). Interestingly, other Urologic Societies have not made formal recommendations on this matter.

The purpose of this study was to survey and define worldwide opioid prescribing patterns after URS. We aimed to define reasons for prescribing opioids after URS and factors that urologists feel impact the need for opioid use in this setting. Types of interventions thought to be most useful to limit opioid prescribing were also explored. We present the following article in accordance with the SURGE reporting checklist (available at http://dx.doi. org/10.21037/tau-20-1121).

\section{Methods}

\section{Ethical statement}

The study was conducted in accordance with the Declaration of Helsinki (as revised in 2013). The study was approved by our institution (University of Vermont) review board (STUDY0000101) and informed consent was taken from all the participants by filling the survey

\section{Survey}

We developed a survey directed to members of the Endourological Society directory. The survey was composed of 12-16 questions targeting practice patterns, frequency of opioids prescription post URS as well as challenges encountered when opioids were not prescribed. In addition, measures thought to be helpful to reduce future opioid prescribing were explored. After developing and reviewing the survey by all the authors, we pilot-tested our survey on a sample of four urologists and finalized the 16-question survey based on that feedback.

\section{Questionnaire administration}

After obtaining our institutional review board approval, the final survey was electronically distributed via REDCap (Nashville, TN, USA) to 2,000 Endourology Society members listed in the 2018-2019 Membership Directory. Respondents were e-mailed a cover letter by the Endourology Society with a hyperlink to the electronic survey. Nonrespondents were e-mailed a reminder cover letter 2 weeks after the first email. Data were organized and secured by the REDCap's proprietary software. No respondent identifiers were collected and no incentives were provided.

\section{Predictor and outcome variables}

Predictor variables included locations of practice, type of practice, endourology fellowship (yes/no), years of practice, and number of ureteroscopies per month.

Our main outcome was routine opioid prescribing post URS which we defined as prescribing opioids post URS more than $50 \%$ of the time. Secondary outcomes were challenges encountered when participants do not prescribe opioids, what they think may lead to the need for opioids and what measures may decrease that.

\section{Statistical analysis}

Data were analyzed using STATA v14 (College Station, TX, USA). Characteristics of participants, opioid prescription patterns post URS, experienced challenges when opioids were not prescribed, patient factors thought to lead to the need for opioids and measures that may decrease the need 
Table 1 Characteristics of participants

\begin{tabular}{|c|c|}
\hline Characteristics & $\mathrm{N}(\%)$ \\
\hline \multicolumn{2}{|l|}{ Location of practice } \\
\hline United States & $57(36.1)$ \\
\hline Canada & $8(5.1)$ \\
\hline Central America & $4(2.5)$ \\
\hline South America & $17(10.8)$ \\
\hline Europe & $36(22.8)$ \\
\hline Asia & $30(18.9)$ \\
\hline Africa & $4(2.5)$ \\
\hline Australia & $2(1.3)$ \\
\hline \multicolumn{2}{|l|}{ Type of practice } \\
\hline Academic Medical Center & $96(60.3)$ \\
\hline Solo Practice Provider & $18(11.3)$ \\
\hline Single Urology Group Practice & $26(16.4)$ \\
\hline Multi-specialty Group & $19(12.0)$ \\
\hline \multicolumn{2}{|l|}{ Fellowship completed } \\
\hline Yes & $105(66.0)$ \\
\hline Fellowship & $\mathrm{N}=105$ \\
\hline Endourology/stone disease & $88(83.8)$ \\
\hline Urologic oncology & $12(11.4)$ \\
\hline Andrology/infertility/erectile dysfunction & $1(1.0)$ \\
\hline Male reconstructive urology/trauma & $2(1.9)$ \\
\hline Female pelvic medicine and reconstructive surgery & $0(0.0)$ \\
\hline Pediatric urology & $1(1.0)$ \\
\hline Transplant & $1(1.0)$ \\
\hline \multicolumn{2}{|l|}{ Years of practice } \\
\hline Less than 5 years & $21(13.2)$ \\
\hline 5 to 10 years & $34(21.4)$ \\
\hline 11 to 20 years & $45(28.3)$ \\
\hline More than 20 years & $59(37.1)$ \\
\hline \multicolumn{2}{|l|}{ Ureteroscopies per month } \\
\hline Less than 10 & $27(17.0)$ \\
\hline Between 10 and 20 & $72(45.3)$ \\
\hline More than 20 & $60(37.7)$ \\
\hline
\end{tabular}

for opioids were all reported with descriptive statistics. We used univariate and multivariate logistic regression to determine risk factors associated with routinely prescribing opioids post URS. Confounding variables were chosen a priori in the multivariate model which included (locations of practice, type of practice, endourology fellowship (yes/no), years of practice, and number of ureteroscopies per month). All tests were two-sided and a $\mathrm{P}$ value of $\leq 0.05$ was considered statistically significant.

\section{Results}

Of the approximately 2000 Endourological Society members who received the email invitation to participate in the survey, 159 completed the survey (8\%).

\section{Characteristics of participants}

The majority of participants were from the US (36.1\%) followed by Europe (22.8\%). Most practiced clinical urology within an academic setting $(60.3 \%)$, practiced for more than 20 years $(37.1 \%)$, and performed between 10 and 20 URS per month (45.3\%). $66 \%$ of respondents completed a fellowship of which endourology was the most common $(83.8 \%)$ (Table 1).

\section{Opioids prescription patterns and challenges experienced when opioids are not prescribed}

Approximately one-quarter (26\%) of respondents reported prescribing opioids more than $50 \%$ of the time (Table 2). The majority $(61.6 \%)$ of respondents prescribed opioids less than $10 \%$ of the time. Thirty-eight percent of the respondents reported having no challenges at all when not prescribing opioids post URS. When challenges were encountered, the most commonly reported were patient calls to clinic for stent related symptoms. Of respondents who reported challenges, these occurred less than half of the time (Table 2).

\section{Factors leading to opioids prescription and measurement to decrease prescription post URS}

The most common factor felt to lead to opioid prescription 
Table 2 Opioids prescription patterns post URS and experienced challenges when opioids are not prescribed

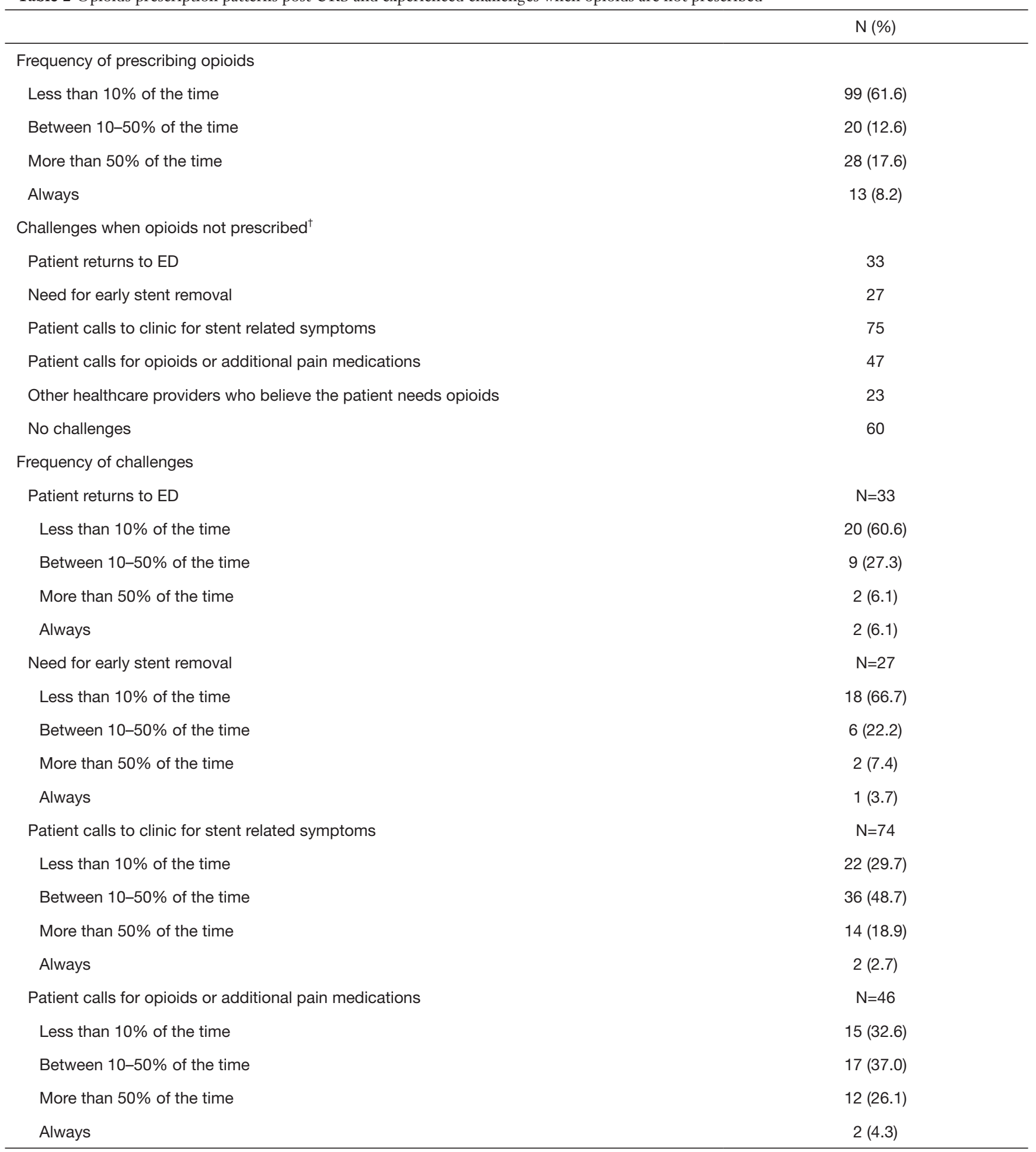

${ }^{\dagger}$, Participants were able to choose multiple choices. URS, ureteroscopy; ED, emergency department. 
Table 3 Patient factors leading to the need for opioids post URS, and measures that may decrease the need for opioids

\begin{tabular}{lc}
\hline & $N(\%)$ \\
\hline Factors leading to the need for opioids ${ }^{\dagger}$ & $32(15.9)$ \\
Patient with mental health diagnoses & $58(28.9)$ \\
Patients with substance abuse disorders & $111(55.2)$ \\
History of needing opioids for previous URS & $109(21.1)$ \\
Measures that may decrease the need for opioids ${ }^{\dagger}$ & 47 (9.1) \\
Pre-op counseling and patient education & $67(13.0)$ \\
Post-op follow-up calls & $116(22.5)$ \\
Printed educational materials & 107 (20.8) \\
NSAIDs use & $70(13.6)$ \\
Adjunct medications (alpha-blockers, anti-cholinergic, phenazopyridine) & \\
\hline
\end{tabular}

${ }^{\dagger}$, Participants were able to choose multiple choices. URS, ureteroscopy.

by respondents was a history of the patient previously taking opioids after URS. Of the measures felt to decrease opioid prescription, the majority of respondents chose NSAIDs use, adjunct medications (alpha-blockers, anti-cholinergic, phenazopyridine), pre-op counseling and patient education (Table 3).

\section{Univariate and multivariate analysis}

On univariate analysis, we found that respondents from the US and Canada were more likely to prescribe opioids routinely post URS [odds ratio (OR) 42.7, $\mathrm{P}<0.001,95 \%$ confidence interval (CI): 12.2-149.2]. Endourologists were less likely to prescribe opioids (OR: $0.5, \mathrm{P}=0.055,95 \% \mathrm{CI}$ : $0.2-1$ ), however, this association did not reach statistical significance. On multivariate analysis, after adjusting for location of practice, type of practice, completion of an endourology fellowship, years of practice, and number of URS per month, we found that respondents from the US and Canada were more likely to prescribe opioids routinely post URS, OR: 87.5, $\mathrm{P}<0.001,95 \%$ CI: 17.3-443.5 (Table 4).

Figure 1 demonstrates percentage of participants who routinely prescribe opioids post URS by location of practice.

\section{Discussion}

The results of our survey highlight several important findings related to opioid prescribing after URS. First, onequarter of our international survey respondents reported prescribing opioids more than half the time after URS. The majority (61.6\%) who prescribed did so less than $10 \%$ of the time. Interestingly, and not surprisingly, there were very obvious differences in prescribing based on geographic location. Compared to the rest of the world, participants from the US and Canada were much more likely to prescribe opioids after URS (Figure 1).

In the US, opioid prescription patterns and outcomes have been studied by several groups. Berger et al., using a private national database of patients undergoing 15 different urologic procedures, found that $51 \%$ filled an opioid prescription after surgery (3). Furthermore, the majority of patients who had a vasectomy and endoscopic kidney stone surgery received opioids (3). Fujii et al. reported on opioid prescribing patterns after various surgical procedures in a single center setting. Using both a retrospective and prospective sample, opioid prescribing was common at $76 \%$ and $92 \%$, respectively. Importantly, the median opioid use was found to be $27 \%$ of the total prescribed and very few (18\%) received proper disposal instructions (13). Brummett et al. used a nationwide insurance data base to look at the incidence of new persistent opioid use after major and minor surgeries. New persistent use was found to be common (5.9-6.5\%) and the incidence did not differ between major and minor procedures. The patients most at risk for persistent use were those with pain conditions, 
Table 4 Univariate and multivariate analysis of provider characteristics associated with prescribing opioids routinely (more than $50 \%$ of the time) post URS

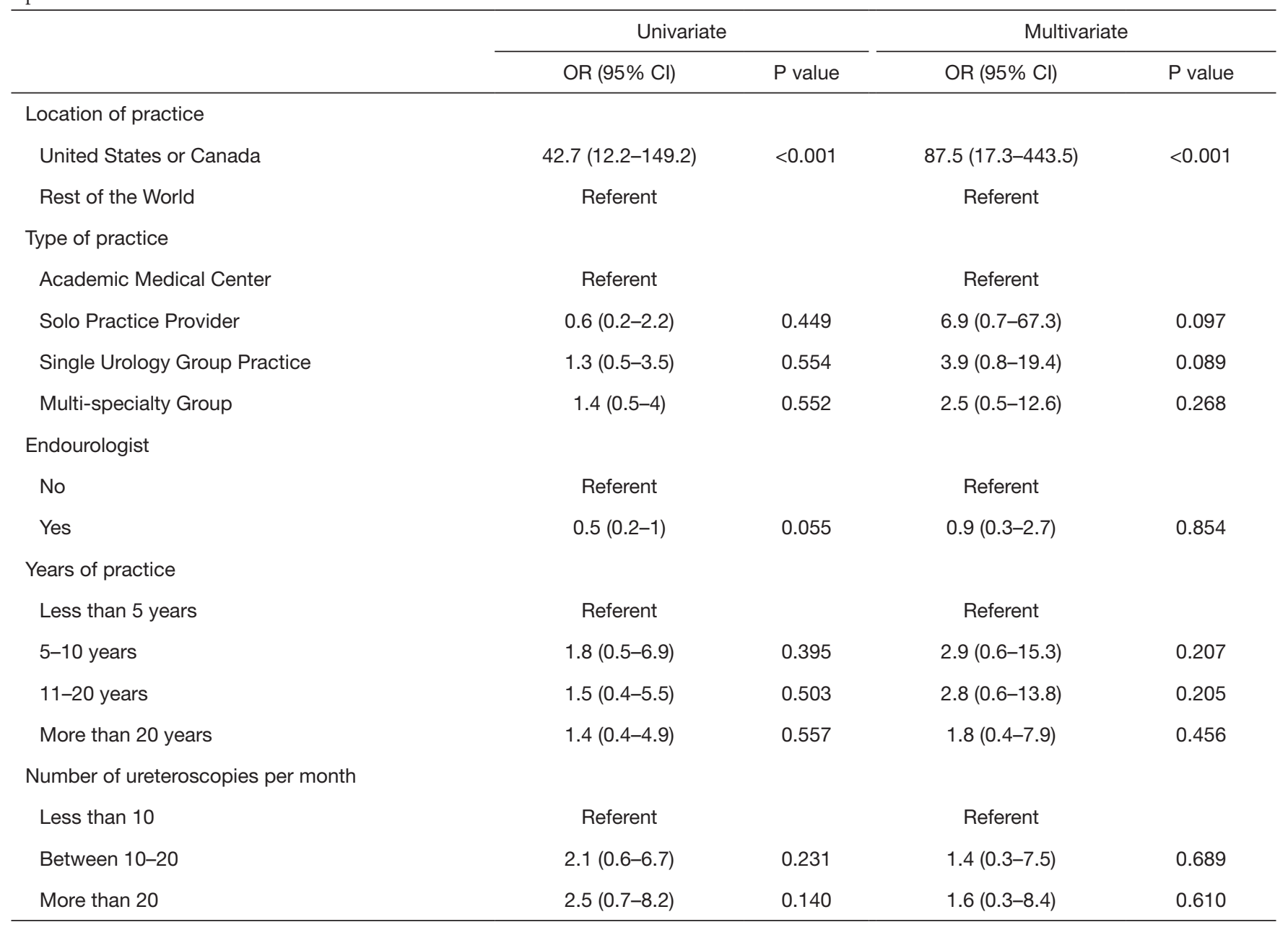

URS, ureteroscopy; OR, odds ratio; Cl, confidence interval.

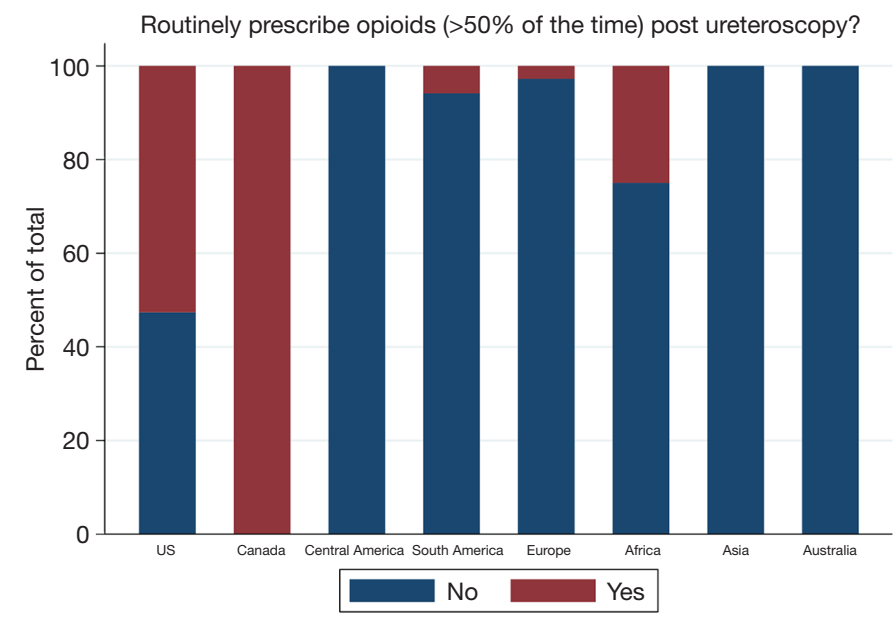

Figure 1 Percentage of participants who routinely prescribe opioids post ureteroscopy by location of practice. 
substance abuse, and mental health disorders (1). The suggestion from this work is that patient factors are likely more influential than postoperative pain in determining postoperative opioid use.

In our survey, we asked urologists to identify patient characteristics thought to increase the likelihood of requiring opioids post operatively and what concerns they have if these medications are not given post procedure. The most common reason that urologists reported that contribute to the need for post URS opioid prescriptions is whether or not the patient needed or received these for previous ureteroscopic intervention. The reasons for this can be surmised to be multi-factorial. There are reports that prior opioid exposure may impact the need for opioids at a later time. Kang et al. retrospectively reviewed patients who underwent URS to identify predictors of receiving additional pain medication after intervention as well as predictors of persistent opioid use defined as use 60 days post-procedure. They found that preoperative opioid exposure including the number of prescriptions, days prescribed and number of different providers was associated with both the need for additional postoperative opioids and long term use (8). Another thought is that stone formers in general have a higher likelihood of longterm opioid use. Shoag et al. used the National Health and Nutrition Examination Survey (NHANES) and found that a self-reported history of kidney stones was associated with current narcotic use (4). In addition, patient history of mental health co-morbidities and substance abuse disorders were also felt by participants in our survey to increase the chances that opioid prescriptions would be needed after URS. Leapman et al. looked at patients undergoing different procedure types for kidney stone treatment in the national Women Veterans Cohort Study (WVCS) and found that patients with a diagnosis of post-traumatic stress disorder PTSD were more likely to receive higher doses of opioids than others in the cohort (14).

The concerns of the urologist when opioids are not given after URS were also addressed in our survey. The most common concern was patient calls to the clinic for stentrelated symptoms. Groups have demonstrated that in their institutional experience, patients who were not given opioids after URS do not in fact call the provider more often than those that do receive them. Large et al. retrospectively reviewed 104 patients who underwent URS with ureteral stent placement in which one group was given opioids and one was narcotic-free. They found no significant differences in patient calls for postoperative pain or need for narcotics between the two groups concluding that a non-opioid approach was safe and effective in their population (10). Similarly, Sobel et al. retrospectively reviewed patients who underwent URS with an intention to avoid opioids whenever possible on discharge. A non-opioid approach was feasible in $75 \%$ of patients and those not receiving opioids did not make more phone calls to clinic for concerning symptoms, require more post-procedure pain medications, or visit the emergency department (ED) more frequently for pain (9). The same group recently presented their updated results at the 2020 AUA annual meeting, which included 391 patients over a span of 3 years and the nonopioid approach was feasible in $91.3 \%$ of patients (15). In addition, Gridley et al. recently implemented an Enhanced Recovery After Surgery (ERAS) protocol prospectively for patients undergoing URS. The rate of opioid prescription on discharge decreased from $93 \%$ to $0 \%$ and similarly there was no difference in postoperative calls for pain or in unscheduled encounters compared to patients discharged pre implementation of the their ERAS protocol (16).

After identifying patient factors that may increase the need for opioids and the concerns of the urologist for not prescribing, the next logical question is what interventions are felt to be useful to limit opioid use in this setting? The most common measures were NSAID use, the use of adjunct medications (alpha-blockers, anticholinergics, phenazopyridine), and pre-op counseling and patient education. Several meta-analyses have reported on the treatment of acute renal colic. Findings support that NSAIDs provide effective analgesia but with fewer adverse events like vomiting and less need for rescue analgesia $(6,7)$.

Patient expectations likely play a major role in how symptoms of pain are managed in the post URS setting. Patients previously receiving opioids for URS may believe that opioids were "needed" in the past and therefore not receiving a prescription goes against the care that they need. For these patients, we feel that this is where education and expectation setting is paramount. While prior opioid use was not incorrect, patients will potentially be more accepting of alternative approaches to pain control when the rationale is carefully explained. Additionally, the general awareness of the detrimental effects of opioids and the epidemic we are facing should lend further support and acceptance. Patients who understand ureteral stent related symptoms and are told what symptoms are concerning warranting medical care likely will call less for the more common routine symptoms. This probably has little to do with the types of medications prescribed. 
Complete avoidance of opioids after URS is probably not feasible nor is it the goal. Most patients however do not require opioids in this setting. The impact of patient education and setting expectations along with a focus on pre-operatively identifying those at risk of needing opioids postoperatively (for example, those with mental health and substance abuse history) have not yet been explored in the literature. This approach may prove to be the most effective way to appropriately decrease post URS opioid prescribing in the future.

\section{Limitations}

Our study is limited by its cross-sectional design with known inherent bias. Our response rate was low (8\%) but common among surveys; as such, there might be a nonresponse bias. The population sample was skewed towards endourologists given our distribution method was through the endourological society, however, 55.4\% of respondents were non-endourologists. In addition, this highlights the opioid prescribing patterns post URS for endourologists who are the most to perform ureteroscopies. We did not account for number of opioid pills prescribed or if prescription patterns differ for participants if their patients were on prior narcotics. Finally, we used a nonvalidated survey which makes it not possible to predict the reproducibility of our study.

Among participants in our survey, nearly one-quarter of urologists prescribe opioids routinely post URS, and US and Canadian urologists were more likely to prescribe routinely compared to the rest of the world. Despite proven feasibility of non-opioid management following URS, many urologists continue to prescribe opioids in this setting. Most important factors felt to reduce opioid prescription post URS were preoperative counseling, and nonsteroidal antiinflammatory drugs use. We believe best practice guidelines by the American and Canadian Urological Associations should be considered to reduce opioid prescribing post URS.

\section{Acknowledgments}

Funding: None.

\section{Footnote}

Reporting Checklist: The authors have completed the SURGE reporting checklist. Available at http://dx.doi. org/10.21037/tau-20-1121

Data Sharing Statement: Available at http://dx.doi. org/10.21037/tau-20-1121

Peer Review File: Available at http://dx.doi.org/10.21037/ tau-20-1121

Conflicts of Interest: All authors have completed the ICMJE uniform disclosure form (available at http://dx.doi. org/10.21037/tau-20-1121). The authors have no conflicts of interest to declare.

Ethical Statement: The authors are accountable for all aspects of the work in ensuring that questions related to the accuracy or integrity of any part of the work are appropriately investigated and resolved. The study was conducted in accordance with the Declaration of Helsinki (as revised in 2013). The study was approved by our institution (University of Vermont) review board (STUDY0000101) and informed consent was taken from all the participants by filling the survey.

Open Access Statement: This is an Open Access article distributed in accordance with the Creative Commons Attribution-NonCommercial-NoDerivs 4.0 International License (CC BY-NC-ND 4.0), which permits the noncommercial replication and distribution of the article with the strict proviso that no changes or edits are made and the original work is properly cited (including links to both the formal publication through the relevant DOI and the license). See: https://creativecommons.org/licenses/by-nc-nd/4.0/.

\section{References}

1. Brummett CM, Waljee JF, Goesling J, et al. New persistent opioid use after minor and major surgical procedures in US adults. JAMA Surg 2017;152:e170504.

2. Bates C, Laciak R, Southwick A, et al. Overprescription of postoperative narcotics: a look at postoperative pain medication delivery, consumption and disposal in urological practice. J Urol 2011;185:551-5.

3. Berger I, Strother M, Talwar R, et al. National variation in opioid prescription fills and long-term use in opioid naïve patients after urological surgery. J Urol 2019;202:1036-43.

4. Shoag JE, Patel N, Posada L, et al. Kidney stones and risk of narcotic use. J Urol 2019;202:114-8.

5. Scales CD Jr, Tasian GE, Schwaderer AL, et al. 
Urinary stone disease: advancing knowledge, patient care, and population health. Clin J Am Soc Nephrol 2016;11:1305-12.

6. Pathan SA, Mitra B, Cameron PA. A systematic review and meta-analysis comparing the efficacy of nonsteroidal anti-inflammatory drugs, opioids, and paracetamol in the treatment of acute renal colic. Eur Urol 2018;73:583-95.

7. Holdgate A, Pollock T. Nonsteroidal anti-inflammatory drugs (NSAIDs) versus opioids for acute renal colic. Cochrane Database Syst Rev 2005;2004:CD004137.

8. Kang C, Shu X, Herrell SD, et al. Opiate exposure and predictors of increased opiate use after ureteroscopy. J Endourol 2019;33:480-5.

9. Sobel DW, Cisu T, Barclay T, et al. A retrospective review demonstrating the feasibility of discharging patients without opioids after ureteroscopy and ureteral stent placement. J Endourol 2018;32:1044-9.

10. Large T, Heiman J, Ross A, et al. Initial experience with narcotic-free ureteroscopy: a feasibility analysis. J Endourol 2018;32:907-11.

11. Koo K, Faisal F, Gupta N, et al. Recommendations for

Cite this article as: Awad MA, Sobel DW, Chew BH, Breyer BN, Plante MK, Sternberg KM. Patterns of opioid prescription post ureteroscopy among members of the Endourological Society. Transl Androl Urol 2021;10(2):851-859. doi: 10.21037/tau-201121 opioid prescribing after endourological and minimally invasive urological surgery: an expert panel consensus. J Urol 2020;203:151-8.

12. Türk C, Neisius A, Petrik A, et al. EAU Guidelines on Urolithiasis. European Association of Urology, 2020.

Available online: https://uroweb.org/wp-content/uploads/ EAU-Guidelines-on-Urolithiasis-2020.pdf (on July 2020).

13. Fujii MH, Hodges AC, Russell RL, et al. Post-discharge opioid prescribing and use after common surgical procedure. J Am Coll Surg 2018;226:1004-12.

14. Leapman MS, DeRycke E, Skanderson M, et al. Variation in national opioid prescribing patterns following surgery for kidney stones. Pain Med 2018;19:S12-8.

15. Awad M, Sobel D, Moring N, et al. MP03-12 Continued feasibility and success of a non-opioid pathway for postoperative pain after ureteroscopy. J Urol 2020;203:e26.

16. Gridley C, Robles J, Calvert J, et al. Enhanced recovery after surgery protocol for patients undergoing ureteroscopy: prospective evaluation of an opioid-free protocol. J Endourol 2020;34:647-53. 